\title{
Acute Physiological Responses and Time- Motion Characteristics of Two Small-Sided Training Regimes in Youth Soccer Players
}

\author{
Stephen V. Hill-HaAs, ${ }^{1}$ Greg J. Rowsell, ${ }^{2}$ Brian T. Dawson, ${ }^{1}$ and Aaron J. Coutrs ${ }^{3}$ \\ ${ }^{1}$ University of Western Australia, Perth, Australia; ${ }^{2}$ South Australian Sports Institute, Kidman Park, Australia; and ${ }^{3}$ School of \\ Leisure, Sport and Tourism, University of Technology, Sydney, Australia
}

\begin{abstract}
Hill-Haas, SV, Rowsell, GJ, Dawson, BT, and Coutts, AJ. Acute physiological responses and time-motion characteristics of two small-sided training regimes in youth soccer players. $J$ Strength Cond Res 23(1): 111-115, 2009-The purpose of this study was to examine the acute physiological responses and timemotion characteristics associated with continuous and intermittent small-sided games (SSGs). The continuous (SSG') regime involved 24 minutes' playing duration (no planned rest intervals), whereas the intermittent regime (SSG') involved $4 \times$ 6-minute bouts with 1.5 minutes of passive planned rest (work:rest ratio 4:1). Both training regimes were implemented across 3 SSG formats, which included games with 2 vs. 2, 4 vs. 4 , and 6 vs. 6 players. Sixteen men's soccer players (mean \pm $S E$ : age $=16.2 \pm 0.2$ years, height $=173.7 \pm 2.1 \mathrm{~cm}$, body mass $=65.0 \pm 2.5 \mathrm{~kg}$, estimated $\dot{V}_{o_{2}} \max =54.8 \pm 0.7$ $\mathrm{ml} \cdot \mathrm{kg}^{-1} \cdot \mathrm{min}^{-1}$ ) participated in the study. Heart rate (HR) was measured every 5 seconds during all SSGs. Global ratings of perceived exertion (RPEs) were recorded immediately after the SSGs using the Borg scale (RPEs, 6-20). Capillary blood samples were drawn at rest and within 5 minutes after the end of each SSG. Time-motion characteristics were measured using portable global positioning system units. There were no significant differences between $S S^{C}$ and $S S G^{\prime}$ for total distance covered or for distance traveled while walking, jogging, or running at moderate speed. However, players covered a significantly greater distance at $13.0-17.9 \mathrm{~km} \cdot \mathrm{h}^{-1}$, a greater total distance at higher running speed, and a greater total number of sprints $\left(>18 \mathrm{~km} \cdot \mathrm{h}^{-1}\right)$ with $S S G^{\prime}$ compared with $\mathrm{SSG}^{\mathrm{C}}$. In contrast, global RPE and \%HRmax were significantly higher in $\mathrm{SSG}^{\mathrm{C}}$ than in SSG'. Both intermittent and continuous SSG training regimes could be used during the season for
\end{abstract}

Address correspondence to Stephen Hill-Haas, hillhaas.stephen@gmail. com.

$23(1) / 111-115$

Joumal of Strength and Conditioning Research

(C) 2009 National Strength and Conditioning Association match-specific aerobic conditioning. However, both training regimes used in this study seem unlikely to provide a sufficient stimulus overload for fully developing $\dot{\mathrm{V}}_{2} \max$.

KEY WorDs continuous training, heart rate, lactate, RPE

\section{INTRODUCTION}

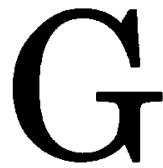
ame-based conditioning using small-sided games (SSGs) has become a popular method of developing specific aerobic fitness for soccer (10). Previous investigators have established that SSGs are both reliable and effective for the technical and tactical development of soccer players $(11,16)$. However, despite the increasing popularity of SSGs, few scientific investigations have examined how the intensity of SSGs can be manipulated to alter the training stimulus.

A number of studies have examined how selected variables (including pitch size, player number, and coach encouragement) can influence SSG training intensity $(14,15,19)$. For example, altering the pitch size has been reported to increase the mean heart rate (HR), blood lactate ( $\mathrm{La}^{-}$), and rating of perceived exertion (RPE) during SSGs $(1,2,16)$. It has also been shown that the mean HR decreases as player numbers increase, when the absolute pitch size constant is held constant $(14,19)$. Indeed, there have recently been several studies that have described the physiological responses to various SSG training formats $(8,11,16)$. However, because of the lack of consistency in SSG design, player fitness, age, ability, level of coach encouragement, and playing rules in each of these studies, it is difficult to make accurate conclusions on the influence of each of these factors separately.

In soccer, SSG training is typically completed in the form of "intervals," as opposed to continuous-duration play, which is more typical of actual game play. Eight weeks of twiceweekly, high-intensity SSG "interval" training (i.e., $4 \times 4$ minutes of SSGs played at an intensity of $90 \%$ HRmax) has been shown to be an equally effective training method for improving aerobic fitness as intensity-matched traditional interval running training (i.e., running around a soccer pitch for $4 \times 4$ minutes at $\mathbf{9 0 - 9 5 \% ~} \mathrm{HRmax}$ ) in well-trained young 
soccer players (10). However, no studies have examined the influence of distributing the dose of SSG training into "interval" bouts rather than longer continuous bouts on physiological responses. Therefore, at present it is unknown whether an SSG "interval" training regime offers any additional benefits above a "continuous" SSG training regime in soccer.

Traditional training methods for developing aerobic power require athletes to work continuously at moderate intensities for longer durations $(4,18)$, such as 60 minutes of continuous cycling at approximately $70 \% \dot{\mathrm{V}}_{2} \max , 5$ times per week (18). However, high-intensity interval training (HIIT) protocols, involving shorter-duration efforts (10 seconds to 4 minutes) at higher intensity, have proven successful at eliciting changes in both aerobic and anaerobic capacity $(7,18)$. Two examples of HIIT protocols involved $4 \times 4$-minute efforts at $90-95 \% \mathrm{HRmax}$, with 3 minutes of recovery (9), whereas another used 7-8 repeats of 20-second efforts at extremely high intensity $\left(\sim 170 \% \dot{\mathrm{V}}_{2}\right.$ max) with 10 seconds of rest (18). However, no previous study has examined the influence of completing SSG in interval compared with continuous training format.

Additionally, no previous studies have examined the timemotion characteristics of SSGs. The time-motion characteristics of individual players represent a method of quantifying the external training load imposed by the training regime. This may include total distance covered and actual time spent at various running speeds. Consequently, the aim of this study was to examine the acute physiological responses and timemotion profiles associated with two distinct SSG training regimes: intermittent $\left(\mathrm{SSG}^{\mathrm{I}}\right)$ and continuous $\left(\mathrm{SSG}^{\mathrm{C}}\right)$. It was also hypothesized that $\mathrm{SSG}^{\mathrm{I}}$ would provide a more intense physiological stimulus and quantitatively greater performance profile compared with $\mathrm{SSG}^{\mathrm{C}}$.

\section{Methods}

\section{Experimental Approach to the Problem}

Few studies have investigated the acute physiological responses and physical performance profiles relating to SSG training regimes $(8,16,19)$. To test the hypothesis, two SSG training regimes were used. The first (SSG) involved a continuous regime of 24 minutes' duration with no planned rest intervals. In contrast, the intermittent (SSGI) regime involved $4 \times 6$-minute bouts with 1.5 minutes of passive planned rest (work:rest ratio $=4: 1$ ), with total playing time (24 minutes) matched to the $\mathrm{SSG}^{\mathrm{C}}$ regime. The two training regimes were implemented across 3 SSG formats, which included 2 vs. 2,4 vs. 4 , and 6 vs. 6 players in each game. The relative pitch size per player remained constant across the 3 different playing formats.

\section{Subjects}

Sixteen men's soccer players (mean $\pm S E$ : age $=16.2 \pm 0.2$ years, height $=173.7 \pm 2.1 \mathrm{~cm}$, body mass $=65.0 \pm 2.5 \mathrm{~kg}$, estimated $\dot{V}_{\mathrm{O}_{2}} \max =54.8 \pm 0.7 \mathrm{ml} \cdot \mathrm{kg}^{-1} \cdot \mathrm{min}^{-1}$ ) participated in the study. All players were members of the same youth (i.e., under 18 years) squad competing in the top-level domestic competition, with a minimum of 8 years' playing experience. A university research ethics committee granted approval for the study. All players and parents were notified of the research procedures, requirements, benefits, and risks before giving informed consent.

\section{Design}

The SSGs were played throughout a 9-week, in-season period in random order, and they were played with consistent coach encouragement at the beginning of normal training sessions (after a standardized 10-minute warm-up), held twice weekly, with at least 48 hours separating each training session. Players were selected on the same teams against the same opponents as often as possible. All players participated in both "interval" and "continuous" SSG training regimes. A major component of preseason conditioning involved SSG training, and, consequently, the players were familiarized with both SSG regimes and all playing formats. The pitch sizes (length $X$ width) used for 2 vs. 2,4 vs. 4 , and 6 vs. 6 games were $28 \times 21 \mathrm{~m}$, $40 \times 30 \mathrm{~m}$, and $49 \times 37 \mathrm{~m}$, respectively, which are similar to the sizes used in previous studies $(14,16,19)$.

\section{Procedures}

The 12-week preseason training period served as a familiarization for all the SSGs and the multistage fitness test (MSFT). This familiarization period also provided an opportunity for the coach to assess each player in terms of passing ability, close ball control, shooting, and game sense skills, using a 5 -point ( 1 = "below average," 5 = "outstanding") Likert scale system. At the conclusion of preseason training, players completed the MSFT and were ranked according to the distance traveled in the MSFT. A composite skill ranking of each player in the squad was generated by the total points achieved for each of the 4 skill components. In an attempt to avoid skill and fitness mismatches and a consequent imbalance in opposing SSG teams, each side was balanced in terms of the individual player's skill and fitness ranking.

Heart rate was measured every 5 seconds during all SSGs (Polar Team Sport System, Polar Electro, Finland). Percentage HRmax (\%HRmax) for each player was determined from MSFT values. Based on the player's peak HR achieved during the MSFT, 4 HR zones were used. These were classified on the basis of \%HRmax. The $4 \mathrm{HR}$ zones used were zone 1 (< 75\% HRmax), zone 2 (75-84\% HRmax), zone 3 (85-89\% HRmax), and zone $4(>90 \% \mathrm{HRmax})$. Both the actual time and percentage of time spent in each zone for each player during all SSGs were calculated.

Global RPEs were recorded immediately after the SSGs using the Borg scale (RPE, 6-20) (5). Capillary blood samples were drawn at rest and within 5 minutes after the end of each SSG. Blood lactate was determined using a blood-gas analyzer (ABL 625 Radiometer, Copenhagen, Denmark).

Time-motion characteristics for every player in each SSG were measured using portable global positioning system units 
(SPI 10, GPSports, Canberra, Australia). The SPI 10 was placed into a harness that positioned the device between the player's shoulder blades. The distance traveled was recorded each second. For data analysis purposes, 4 speed zones were selected: speed zone 1 (standing and walking, $0-6.9 \mathrm{~km} \cdot \mathrm{h}^{-1}$ ), speed zone 2 (jogging, $7.0-12.9 \mathrm{~km} \cdot \mathrm{h}^{-1}$ ), speed zone 3 (moderate-speed running, 13.0-17.9 $\mathrm{km} \cdot \mathrm{h}^{-1}$ ), and speed zone 4 (higher-speed running, $>18.0 \mathrm{~km} \cdot \mathrm{h}^{-1}$ ). A "sprint activity ratio" was also calculated to reflect how "active" a particular player was during the course of an SSG. The ratio was calculated by dividing the elapsed time gap between the final and first sprints by the total SSG time and then by multiplying this by the total number of sprints $>18.0 \mathrm{~km} \cdot \mathrm{h}^{-1}$. The ratio will penalize both the player who completes a cluster of sprints infrequently and the player who only completes a few single sprints that happen to be evenly distributed throughout the SSG. The ratio will favor a player who makes frequent clusters of sprints throughout the SSG.

\section{Statistical Analyses}

The data are reported as mean $\pm S E$. A 1-way repeatedmeasures analysis of variance was used on each dependent variable including $H R, R P E$, blood lactate, and various timemotion characteristics. The independent variables were the 2 different SSG regimes used: intermittent and continuous. When significant interaction effects were found, Scheffe post hoc comparisons were applied. Statistical significance was set at $p \leq 0.05$. The statistical analyses were performed using the software package STATISTICA (version 6.0, Statsoft, Tulsa, Okla).

\section{Results}

There were no significant differences between $\mathrm{SSG}^{\mathrm{C}}$ and $\mathrm{SSG}^{\mathrm{I}}$ for total distance covered or for distance traveled while walking, jogging, or running at moderate speed. However, players covered a significantly greater distance at 13.0$17.9 \mathrm{~km} \cdot \mathrm{h}^{-1}$ with SSG ${ }^{\mathrm{I}}$ compared with $\mathrm{SSG}^{\mathrm{C}}$ (Table 1 ).

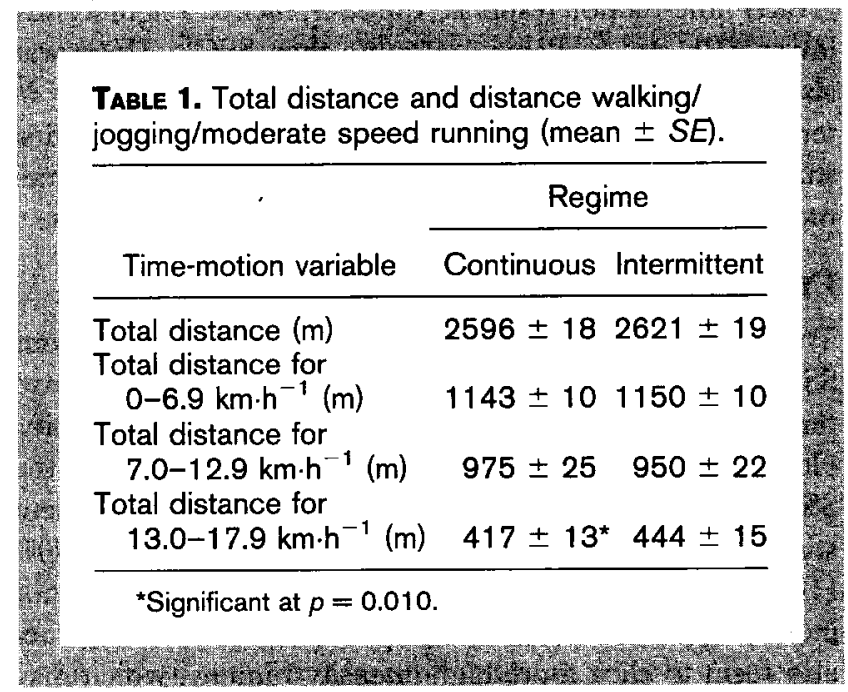

The players also covered a significantly greater total distance at higher running speeds $\left(>18 \mathrm{~km} \cdot \mathrm{h}^{-1}\right)$ during $\mathrm{SSG}^{\mathrm{I}}$ compared with SSG $^{\mathrm{C}}$. Additionally, players completed a significantly greater total number of sprints $\left(>18 \mathrm{~km} \cdot \mathrm{h}^{-1}\right)$ during SSG $^{\mathrm{I}}$ compared with $\mathrm{SSG}^{\mathrm{C}}$. There was also a significantly higher sprint activity ratio for $\mathrm{SSG}^{\mathrm{I}}$ compared with SSG $^{\mathrm{C}}$ (Table 2).

The acute physiological and perceptual responses are shown in Table 3. Global RPE and \%HRmax were significantly higher in $\mathrm{SSG}^{\mathrm{C}}$ than in SSG. However, there were no significant differences in any of the other variables (Table 3).

\section{Discussion}

The aim of this study was to examine the acute physiological responses and time-motion profiles associated with intermittent and continuous soccer SSG training regimes. Although many studies have reported on time-motion characteristics of soccer match play $(7,15)$, to our knowledge, no study has reported on the time-motion characteristics of SSG training.

The intermittent training regime elicited significantly more moderate- and higher-speed running (13-17.9 and $>18$ $\mathrm{km} \cdot \mathrm{h}^{-1}$, respectively) compared with the continuous regime. The frequency of repeated sprint efforts was also significantly higher in SSG. One possible explanation for the greater

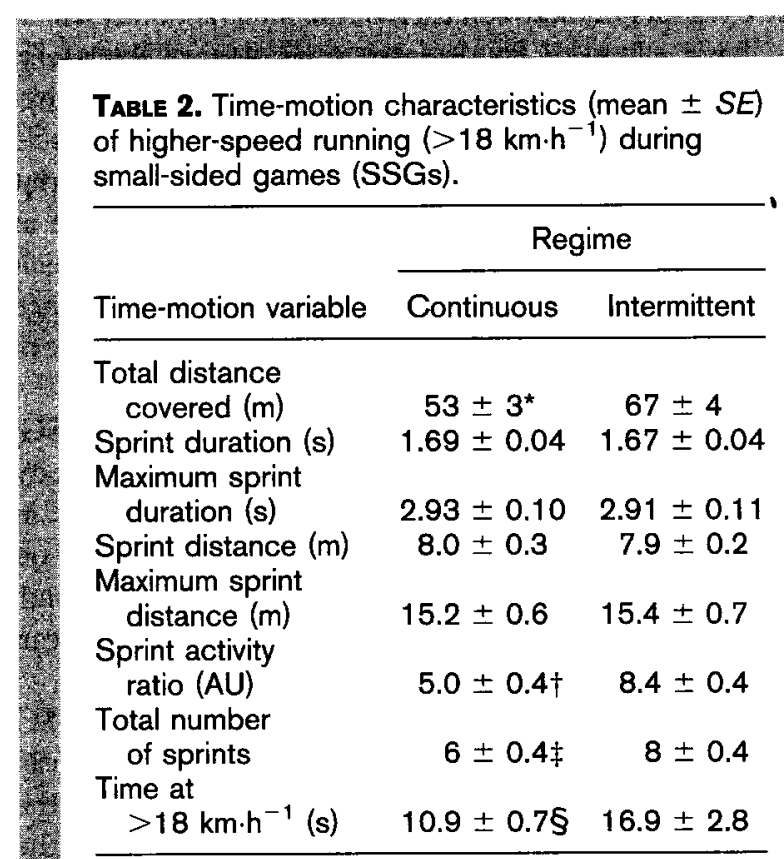

Sprint activity ratio was calculated by dividing the elapsed time gap between the final and first sprints by the total SSG time, and multiplying this by the total number of sprints $>18.0 \mathrm{~km} \cdot \mathrm{h}^{-1}$.

*Significant difference between regimes, $p=0.007$.

$\dagger p=0.001$.

$\Varangle p=0.002$

$\S p=0.044$. 


\begin{tabular}{|c|c|c|}
\hline $\begin{array}{l}\text { TABLE 3. Physiolog } \\
\text { characteristics of } s \\
\text { (mean } \pm S E \text { ). }\end{array}$ & $\begin{array}{l}\text { and percept } \\
\text { ll-sided game }\end{array}$ & (SSGs) \\
\hline \multirow{2}{*}{$\begin{array}{l}\text { Physiological or } \\
\text { perceptual variable }\end{array}$} & \multicolumn{2}{|c|}{ Regime } \\
\hline & Continuous & Intermittent \\
\hline$\% H R \max$ & $87 \pm 1^{\star}$ & $84 \pm 1$ \\
\hline $\begin{array}{l}\text { Time at }<75 \% \\
\text { HRmax (min) }\end{array}$ & $1.52 \pm 0.25$ & $0.83 \pm 0.22$ \\
\hline $\begin{array}{l}\text { Time at } 75-84 \% \\
\text { HRmax (min) }\end{array}$ & $4.85 \pm 0.56$ & $4.92 \pm 0.49$ \\
\hline $\begin{array}{l}\text { Time at } 85-89 \% \\
\text { HRmax (min) }\end{array}$ & $7.45 \pm 0.61$ & $7.84 \pm 0.48$ \\
\hline $\begin{array}{l}\text { Time at }>90 \% \\
\text { HRmax (min) }\end{array}$ & $10.22 \pm 0.62$ & $10.42 \pm 0.56$ \\
\hline $\begin{array}{l}\text { Blood } \\
\mathrm{La}^{-}\left(\mathrm{mmol} \cdot \mathrm{L}^{-1}\right)\end{array}$ & & $4.8 \pm 0.3$ \\
\hline $\operatorname{RPE}(A U)$ & $12.3 \pm 0.2 \dagger$ & $11.6 \pm 0.2$ \\
\hline \multicolumn{3}{|c|}{$\begin{array}{l}\text { HRmax }=\text { maximum heart rate; RPE }=\text { rating of } \\
\text { perceived exertion. } \\
\text { *Significant difference between regimes, } p=0.001 \text {. } \\
\dagger p=0.02 \text {. }\end{array}$} \\
\hline
\end{tabular}

frequency of sprint activity during $\mathrm{SSG}^{\mathrm{I}}$ was the additional passive rest period between each of the interval bouts, which may have allowed for greater physiological recovery, including $\mathrm{PCr}$ resynthesis and removal of metabolic byproducts including the accumulation of potassium in muscle interstitium $(3,6)$. Despite the greater frequency of sprint activity during $\mathrm{SSG}^{\mathrm{I}}$, there was no significant difference in sprint distance or duration between the 2 regimes, which is supported by a recent finding that both sprint distance and exercise:rest ratio independently influence the rate of fatigue (12).

Although there was no significant difference between $S_{S G}{ }^{\mathrm{I}}$ and $\mathrm{SSG}^{\mathrm{C}} \mathrm{La}^{-}$response, the $\mathrm{SSG}^{1} \mathrm{La}^{-}$values in this study are similar to those reported for intermittent SSGs in previous studies $(1,16)$. No other studies have reported on the $\mathrm{La}^{-}$response associated with continuous SSGs, and, consequently, no comparison is possible. The range of mean $\mathrm{La}^{-}$values observed during matches is $2-10 \mathrm{mM}$ (3).

The RPE values measured during the intermittent SSG training regime in this study were lower than previously reported values $(1,17)$. A possible explanation for the decreased RPE in the present study compared with the findings of Sampaio et al. (17) $(4 \times 6$ minutes with 90 seconds of recovery vs. $2 \times 3$ minutes with 90 seconds of recovery) relates to the longer intermittent playing durations applied in the present study. These results suggest that higher RPE values are associated with very short intermittent playing durations, which implies that players are able to play at very high intensity (substantially above match intensity) for only short durations during intermittent SSG training.
Consequently, longer intermittent playing durations are perceived as less intense by the players. In the present study, $\mathrm{SSG}^{\mathrm{I}}$ elicited a significantly lower RPE than $\mathrm{SSG}^{\mathrm{C}}$. Although comparisons with other studies are not possible, one possible reason may be the additional planned rest periods between intermittent bouts allowing for greater recovery.

The $\mathrm{SSG}^{\mathrm{C}}$ training elicited a significantly higher \%HRmax response compared with $\mathrm{SSG}^{\mathrm{I}}$. A possible explanation is that the additional rest period between the work bouts during $\mathrm{SSG}^{\mathrm{I}}$ enabled the players to start subsequent work bouts with a lower \% HRmax, resulting in an overall lower \% $\mathrm{HRmax}$ for SSG $^{\mathrm{I}}$. The $\% \mathrm{HRmax}$ values for SSG ${ }^{\mathrm{I}}$ attained in this study are similar to those previously reported for small-format ( 2 vs. 2 and 3 vs. 3 ) intermittent games training $(1,14,17)$. The $\% \mathrm{HRmax}$ values for $\mathrm{SSG}^{\mathrm{C}}$ attained in this study, however, are similar to those reported for larger-format ( 5 vs. 5 and 6 vs. 6 ) intermittent games training $(13,14,16)$. However, comparisons are more difficult because the majority of studies applied very-short-duration playing bouts during SSG $^{\mathrm{I}}$ (90 seconds to 3 minutes, compared with 6 minutes). To our knowledge, only one previous study (2) compared a 30minute continuous SSG with a series of intermittent SSGs using a variety of work:rest ratios and (intermittent) playing bouts ranging from 30 seconds to 8 minutes. In contrast to this study, the continuous SSG was reported to have a lower HR response compared with the intermittent games. Unfortunately, comparisons with this study are made more difficult because average HR was reported instead of $\% H R m a x$, and the data were only reported for one subject.

In summary, the intermittent training regime in this study elicited significantly more moderate- and higher-speed running (13-17.9 and $>18 \mathrm{~km} \cdot \mathrm{h}^{-1}$, respectively) compared with the continuous regime. Furthermore, the frequency of repeated sprint efforts was also significantly higher in SSG. However, despite the greater frequency of sprint activity during $S_{S G}$, there was no significant difference in sprint distances or duration between the 2 regimes. Furthermore, although the intermittent games caused a significantly greater time-motion response, the $S S G^{\mathrm{I}}$ elicited a significantly lower RPE and \%HRmax response than $S_{S G}$. There was also no significant difference between $\mathrm{SSG}^{\mathrm{I}}$ and $\mathrm{SSG}^{\mathrm{C}}$ in terms of $\mathrm{La}^{-}$response. To our knowledge, this is the first study to compare acute physiological responses and timemotion characteristics of 2 distinct SSG training regimes.

\section{Practical Applications}

The results indicate that both intermittent and continuous SSG training regimes could be used during the season to provide a conditioning stimulus similar to match intensity. However, both the continuous and interval formats of the SSGs used in this study seem unlikely to provide a sufficient stimulus overload because a training intensity $(>90 \%$ HRmax) has previously been suggested to be optimal for increasing $\dot{\mathrm{V}}_{2}$ max in soccer players $(7,9)$. The $\mathrm{SSG}^{\mathrm{I}}$ games also seem to elicit more high-intensity running, which may 
be useful for developing fitness requiring repeated sprint efforts with short recovery. The intermittent SSGs were also perceived by the players to be easier, which may suggest greater compliance among players if this training is used frequently. Finally, another potential benefit is that, by design, SSG ${ }^{I}$ training allows for coaching input during the passive rest periods. This may facilitate improved skill transfer and decision making between SSG training and match situations. However, more research is needed to investigate the extent of skill involvement and the quality of skilllearning outcomes.

\section{ACKNOWLEDGMENTS}

We would like to thank S. Potter and J. Lorreto of Cockburn City S.C. for their administrative and technical/coaching support, respectively, as well as the 2005 U18 Premier League squad players for their steadfast commitment and effort. We would also like to thank Polar Electro Oy (Finland) for sponsoring the Polar Team Sport System heart rate monitors and software. The results of the present study do not constitute endorsement of the product by the authors or the journal.

\section{REFERENCES}

1. Aroso, J, Rebelo, AN, and Gomes-Pereira, J. Physiological impact of selected game-related exercises. J Sports Sci 22: 522, 2004.

2. Balsom, PD, Lindholm, T, Nilsson, J, and Ekblom, B. Precision Football. Kempele, Finland: Polar Electro Oy, 1999.

3. Bangsbo, J, Laia, M, and Krustrup, P. Metabolic response and fatigue in soccer. Int J Sports Physiol Perform 2: 111-127, 2007.

4. Bangsbo, $\mathrm{J}$ and Michalak, L. Assessment of the physiological capacity of elite soccer players. In: Science $\xi^{\circ}$ Football $I V$. W. Spink, T. Reilly, and A. Murphy, eds. London: Routledge, 2002. pp. 53-62.

5. Borg, G. Borg's Perceived Exertion and Pain Scales. Champaign: Human Kinetics, 1998.

6. Gaitanos, GC, Williams, C, Boobis, LH, and Brooks, S. Human muscle metabolism during intermittent maximal exercise. $J$ Appl Physiol 75: 712-719, 1993.
7. Helgerud, J, Engen, LC, Wisloff, U, and Hoff, J. Aerobic endurance training improves soccer performance. Med Sci Sports Exerc 33: 1925-1931,2001.

8. Hill-Haas, S, Coutts, A, Rowsell, G, and Dawson, B. Variability of acute physiological responses and performance profiles of youth soccer players in small-sided games. J Sci Med Sport 11: 487-490, 2008.

9. Hoff, J, Wisloff, U, Engen, LC, Kemi, OJ, and Helgerud, J. Soccer specific aerobic endurance training. $B r J$ Sports Med 36: 218-221, 2002.

10. Impellizzeri, FM, Marcora, SM, Castagna, C, Reilly, T, Sassi, A, Iaia, $M$, and Rampinini, E. Physiological and performance effects of generic versus specific aerobic training in soccer players. Int $J$ Sport Med 27: 483-492, 2006.

11. Little, T and Williams, AG. Suitability of soccer training drills for endurance training. $J$ Strength Cond Res 20: 316-319, 2006.

12. Little, $T$ and Williams, AG. Effects of sprint duration and exercise: rest ratio on repeated sprint performance and physiological responses in professional soccer players. J Strength Cond Res 21: 646648, 2007.

13. Little, T and Williams, AG. Measures of exercise intensity during soccer training drills with professional soccer players. J Strength Cond Res 21: 367-371, 2007.

14. Owen, A, Twist, C, and Ford, P. Small-Sided Games: The physiological and technical effect of altering pitch size and player numbers. Insight 7: 50-53, 2004.

15. Rampinini, E, Coutts, A, Castagna, C, Sassi, R, and Impellizzeri, F. Variation in Top Level Soccer Match Performance. Int J Sport Med 28: 1-7, 2007.

16. Rampinini, E, Impellizzeri, F, Castagna, C, Abt, G, Chamari, K, Sassi, A, and Marcora, SM. Factors influencing physiological responses to small-sided soccer games. J Sports Sci 25: 659-666, 2006.

17. Sampaio, J, Garcia, G, Macas, V, Ibanez, J, Abrantes, C, and Caixinha, P. Heart rate and perceptual responses to $2 \times 2$ and $3 \times 3$ small-sided youth soccer games. J Sports Sci Med 6(Suppl. 10): 121-122, 2007.

18. Tabata, I, Nishimura, K, Kouzaki, M, Hirai, Y, Ogita, F, Miyachi, M, and Yamamoto, K. Effects of moderate-intensity endurance and high-intensity intermittent training on anaerobic capacity and $\dot{V}_{\mathrm{o}_{2}}$ max. Med Sci Sports Exerc 28: 1327-1330, 1996.

19. Williams, $K$ and Owen, $A$. The impact of player numbers on the physiological responses to small sided games. J Sports Sci Med 6(Suppl. 10): 100, 2007. 